\title{
Forming and canceling everyday intentions: Implications for prospective remembering
}

\author{
PAUL M. DOCKREE and JUDI A. ELLIS \\ University of Reading, Reading, England
}

\begin{abstract}
The intention superiority effect(ISE) is characterized by faster response time to task material intended for future performance than to neutral material with no associated intention or material that is linked to a canceled intention. The existence of the ISE has been explored here under naturalistic conditions in which participants self-initiate an intention that is of personal relevance to them. Participants were required to remember prospective tasks that were presented under the guise of preparatory tasks for the next participant. After encoding a pair of tasks, they were informed that one task no longer needed to be performed. Subsequent lexical decision data exhibited the expected effect of faster response time for intended items than for canceled items (experimental groups in Experiments 1A and 1B). No differences in response time were observed between two sets of canceled items (control group in Experiment 1A). When an intention coexisted with the expectation that a written description of the task would be available, no reliable difference in latencies for these items and canceled items was observed (control group in Experiment 1B). The results are discussed in terms of facilitatory and inhibitory processes that may allow us to contend with many intentions in everyday scenarios.
\end{abstract}

Prospective remembering requires the operation of a variety of cognitive processes to enable an intention to be postponed until a suitable opportunity for its execution (cf. Dobbs \& Reeves, 1996; Ellis, 1996). In this paper, we focus on examining processes underlying the encoding and accessibility of an intention prior to its retrieval/execution. In particular, we explore the generality of the intention superiority effect-the finding that information intended for future performance is more accessible from memory than neutral information with no associated intentionality (Goschke \& Kuhl, 1993; Marsh, Hicks, \& Bink, 1998).

The intention superiority effect has been demonstrated using a delayed intention paradigm, introduced by Goschke and Kuhl (1993). Their participants were required to memorize short descriptions of four everyday activities or "scripts," presented in pairs. Each script comprised simple action phrases; for example, the script setting the dinner table is made up of the component actions spread the table cloth, distribute the cutlery, and so forth. After having learned a pair of scripts, the participants were told that at a later point they would have to execute one of the scripts or observe the experimenter execute one. These instructions lead to the identification of a prospective script (to observe or to perform an activity). The second script of the pair was described as neutral insofar as it had no associated inten-

This research was supported by the award of a postgraduate research studentship to the first author from the Economic and Social Research Council: Award R00429834382.Correspondence concerning this article should be addressed to P. M. Dockree, Department of Psychology, University of Reading, Earley Gate, Reading RG6 6AL, England (e-mail: p.m.dockree@ reading.ac.uk). tion to perform or observe performance. Following these instructions, the participants undertook a recognition test in which shorter response latencies were observed for tobe-enacted script words than for neutral script words. In a separate experimental block, with a second pair of scripts, no such difference was observed between to-be-observed script words and the corresponding neutral words.

Using Anderson's (1993) adaptive control of thought (ACT-R) model, Goschke and Kuhl (1993) suggested that the observation that intentions appear to decay more slowly than other memory contents could be ascribed to their representation as subthreshold source nodes in longterm memory. Anderson's model proposes that goals for immediate action in working memory are represented as source nodes that maintain their activation level without rehearsal. Importantly, in this regard, Goschke and Kuhl observed a reliable intention superiority effect across four separate experiments in which they claim to have excluded "routine encoding factors" that might have strategically changed the representation of the to-be-enacted script.

In an extension of this paradigm, using similar script materials, Marsh, Hicks, and Bink (1998) argued that purer measures of activation can be inferred from the lexical decision test (LDT) and, accordingly, observed the occurrence of the intention superiority effect across four experiments. They also suggested that to explain of this effect in terms of heightened and persistent activation of intentions would be somewhat counterintuitive, given that we have to deal with multiple intentions during the course of the day and would therefore need to activate and inhibit them throughout this period. They proposed a more realistic explanation, suggesting that intended information is reprocessed rapidly or more efficiently as a consequence of 
an intentional marker. This marker provides an internal context cue that is triggered once its retrieval conditions have been satisfied (cf. Norman \& Shallice, 1986; Shallice \& Burgess, 1991). In support of this, Marsh, Hicks, and Bink demonstrated that latencies to completed (performed) script material were longer than those to comparable neutral material, suggesting that once an intention has been carried out, its content is less available or accessible.

Marsh, Hicks, and Bryan (1999) have adapted the delayed intention paradigm to correspond to a more realworld scenario by asking participants to encode numerous unrelated activities (unrelated verb-the-noun phrases). This manipulation was based on the observation that people often have multiple intended goals over the course of a day; for example, the intention to post a letter, go to the cash point, and make a phone call today would constitute unrelated intended actions. Using this modified design, they replicated the intention superiority effect for lexical decision latencies. Importantly, therefore, they demonstrated that conditions designed to mimic one characteristic of everyday intentions translate to differences in activation as measured by the delayed intention paradigm.

In view of the shifting moment-to-moment demands placed on an intentional system (cf. Marsh, Hicks, \& Landau, 1998), it is important to examine both the relevance of the ISE to everyday planned actions and the usefulness of the current paradigm in a more naturalistic context wherein intentions are often self-initiated. Everyday intentions are undoubtedly goal directed, yet the script material employed in previous experiments, although possessing a goal-orientated structure, has lacked any selfreference or purpose and may thereby have acquired an arbitrary status. In defense of this criticism, Goschke and Kuhl (1993) suggested that activities with little personal relevance to participants allow for a purer measure of intention superiority because the influence of motivationally charged material is minimized. They believe that "the effect [the ISE] seems to be a specific consequence of the intentional format of an action representation." (Goschke \& Kuhl, 1993, p. 1223).

Notwithstanding the preceding argument, Kuhl (1985) has acknowledged that the propositional structure of an intention should include a component linking the agent of the intended action to some aspect(s) of the self. This feature may have important consequences for encoding and retention. Rogers, Kuiper, and Kirker (1977), for example, have demonstrated superior recall for material rated under a selfreference task over information encoded under structural, phonemic, or semantic orienting tasks (see also Klein, Loftus, \& Burton, 1989; Klein \& Kihlstrom, 1986). They have argued that self-referent material activates a superordinate schema of the self that imparts a degree of richness and fullness to the encoding process. If self-reference plays a salient role as an effective encoding variable, then it is important to examine the activation level or accessibility of intentions with necessary and meaningful consequences.
The contribution of self-referent as well as self-initiated intentions has not yet been examined with respect to intention superiority. Schaefer, Kozak, and Sagness (1998), however, have devised a novel task that enabled them to incorporate these factors in a study of intention retrieval and performance. They introduced prospective tasks under the guise of preparatory tasks for the next participant. In this way, the tasks were interpreted as genuine requests from the experimenter, with a real purpose and disguised by a cover task. In addition, because the experimenter explained that he/she would not be available at the end of the experiment, the expectation that performance of the prospective tasks would be uncued (i.e., reliant on self-initiation processes) was introduced.

The primary motivation for the experiments reported here was to create conditions more typical of everyday planned actions by adapting the naturalistic experimental conditions introduced by Schaefer et al. (1998), in order to investigate the occurrence of the ISE for self-relevant intentions that require self-initiated retrieval.

\section{Experiments 1A and 1B}

In both experiments, we employed the basic design of Goschke and Kuhl's (1993) and Marsh, Hicks, and Bink's (1998) experiments in that we used experimental (to-beenacted) and control (neutral) scripts. The conditions in both experiments were identical, but a different control group was used in each.

The general method for the experimental groups was as follows. The participants were initially asked to encode two tasks that they would need to perform later in the study; poststudy questioning established that these tasks were interpreted as genuine requests from the experimenter. They were also asked to undertake a series of culture fair tests (the cover task). At a later point, the experimenter explained that one of the intended tasks was no longer necessary (owing to time constraints) and that only one task need be carried out. In a subsequent LDT, response times to intended and nonintended words were compared. After completion of the LDT, the participants continued with the culture fair tests for a set period before carrying out, uncued, the appropriate intended task prior to leaving the room. In summary, in these two experiments, we investigated the relative activation/accessibility of maintained and canceled intentions that have self-relevance and that require self-initiated retrieval processing.

\section{METHOD}

\section{Participants}

Eighty undergraduates from the University of Reading volunteered for either Experiment 1A or Experiment 1B (Experiment 1A, $N=32$; Experiment 1B, $N=48$ ), in exchange for either course credit or a small payment. All participants were between 18 and 26 years of age. There were four conditions (one experimental and one control in each of Experiments 1A and 1B). In Experiment 1A, 16 participants were allocated to the experimental group and a further 
16 to the control group. In Experiment 1B, there were 24 participants in each condition.

\section{Materials}

The preparatory tasks consisted of two activities, each comprising four propositions. Both activities, detailed in Appendix A, had a heading and a sequence of action phrases. The structure of the tasks does not conform exactly to the verb and noun structure used in previous investigations (cf. Goschke \& Kuhl, 1993). Here, in keeping with a more naturalistic scenario, more explanatory action phrases were used, for example, "reverse the sign on the door when you leave." Only the key noun and verb from each action phrase, which represented the core action components, were selected as task items. In the case of the latter example, the words reverse and sign were selected. The LDT (LDT) was constructed from three types of items: task words, nontask words, and nonwords. Sixteen nouns and verbs from both activities were used for the task words, and a further 16 unrelated nouns and verbs formed the nontask words. Nonwords consisted of 32 pronounceable letter strings. Task and nontask words were matched for word frequency (Francis, $\mathrm{Ku}$ čera, \& Mackie, 1982) and syllabic length. Nonwords were matched for syllabic length to the task and nontask words. A further 12 items ( 6 words and 6 nonwords) were used as buffer items that preceded these 64 critical items in the LDT. Complete randomization of critical items was under software control.

The preparatory tasks and the LDT were embedded in a series of measures taken from the Culture Fair Intelligence tests (Cattell \& Cattell, 1960). Tests 1, 2, and 3 from Scale 2, Form A were selected and presented in one of three booklets (CFT-1, CFT-2, and CFT-3). These three booklets were presented, as clearly labeled separate sections, in an experimental folder entitled "The Perception of Relationships in Shapes and Figures." Each test was preceded by a brief explanation of how to carry it out, and the experimental folder included instructions for when and how to initiate and carry out the LDT. (Pictorial tests were selected to avoid lexical interference following encoding of the preparatory tasks.) All the task materials were in clear view and in easy reach of the participants, and the initial arrangement of the room was consistent across participants (see Appendix B).

\section{Design and Procedure}

The participants were told that the experiment was designed to examine the perception of relationships in shapes and figures. It was made clear to them that the experimenter would have to leave at the end of the first Culture Fair Test (CFT-1) in order to conduct a different experiment with another participant. The prospective materials, described earlier, were introduced under the guise of preparatory tasks (consistent with Schaefer et al., 1998).

The preparatory tasks were described in the following instructions: "You are the first participant to be tested in this particular experimental condition. Because I am testing another participant at the same time I probably will not be here when you are finished with the experiment. Would you please do two things before you leave in preparation for the next participant, who is undertaking a different experiment from you." They were presented on a laminated sheet, one above the other, with the order of presentation counterbalanced across participants. To maintain the deception, it was explained that another participant, whom the experimenter was allegedly testing in another room, was also required to perform these preparatory tasks. Consequently, the experimenter needed to remove the sheets on which the task instructions were printed when he left the room. This deception was necessary in order to encourage participants to memorize both preparatory tasks without drawing attention to the relevance of this material to the experimental design. All participants were under the impression that they were the first to take part in this particular experimental condition. (In keeping with Schaeffer et al. (1998), the assignment of each participant as the first in the study was employed to induce similar levels of motivation across participants.)
The participants were given 2 min to memorize the preparatory tasks. (Unlike Goschke \& Kuhl and Marsh et al., we did not insist on criterion learning, because we thought that this would discredit the experimental deception). The experimenter left the room during this period under the pretense that he needed to attend to a concurrent experiment in another room. On his return, the sheets describing the preparatory tasks were removed, and the participant was given the experimental folder. Instructions explaining how to undertake all further tests were included in the folder, and the experimenter went through these with the participant and clarif ied any queries. The allotted time for CFT-1 was $2 \mathrm{~min}$, and the experimenter remained in the room monitoring the time. After completion of CFT- 1 and before the initiation of the LDT, the experimenter stated, "Because of the time available you no longer need to prepare [Task 1], please only prepare [Task 2]." In this way, the intentional status for one preparatory task was removed or canceled but maintained for the other. The assignment of intended versus canceled status to preparatory tasks was counterbalanced across participants.

Experiments 1A and 1B differed with respect to the independent control groups used. The different prospective instructions given to the control groups were as follows.

Experiment 1A. For this control group, it was maintained that "due to time constraints, you no longer need to prepare [Task 1] or [Task 2]." Therefore, the intentional status of both preparatory tasks was canceled.

Experiment 1B. In this control group, the intentional status of one preparatory task was canceled (as in both experimental groups) but, unlike in an experimental group, the participants were told after encoding that they could refer to the preparatory task sheet at the end. In this way, it was intended that the participants would place less reliance on maintaining an internal representation of the intended material.

Following instructions to maintain (or cancel) the appropriate intention(s) and the subsequent departure of the experimenter, the participants initiated performance of the LDT. Instructions for carrying out this task were included in the experimental folder. On completion of the LDT, the participants returned to the experimental booklet and completed CFT-2 and CFT- 3 in the allotted time of 8 min. A final page in the booklet required the participants to perform two final "finishing" tasks: to put the answer booklet in the box provided, and to pick up the "research panel questionnaire." The final sentence, consistent with Schaefer et al. (1998), was worded as follows: "If the experimenter has not returned, having completed the requested tasks, you are free to leave." The term requested tasks could refer to the final finishing tasks or the prospective task; this was purposely ambiguous. Upon leaving the room, each participant was unexpectedly stopped by the experimenter and asked to complete a short questionnaire. The participants answered (1) what they thought the purpose of the experiment was, (2) whether they had suspected that the preparatory tasks were relevant to the experiment, and (3) whether they had recognized any of the words from the preparatory tasks during their performance of the LDT.

Finally, the participants were debriefed as to the purpose of the experiment and, in the experimental conditions and control condition of Experiment 1B, the experimental room was checked to see whether the appropriate preparatory task had been carried out as had been instructed. In the control condition of Experiment 1A, the room was checked to see whether both tasks had been correctly canceled.

\section{RESULTS}

Following Marsh, Hicks, and Bink (1998), all incorrect responses were removed from analyses of the lexical decision data and latencies beyond 3 standard deviations of each participant's mean response were also excluded. In Experiment 1A, this resulted in the removal of $4.69 \%$ of all responses as errors and $0.55 \%$ as outliers. It was nec- 
essary to replace one participant in the experimental group who mistakenly carried out both preparatory tasks and thus failed to cancel the appropriate one. In Experiment 1B, $4.92 \%$ of responses were errors and $1.78 \%$ of the responses were classified as outliers, in accordance with criteria above. These responses were excluded from the analyses. In addition, it was necessary to replace 2 participants in the control group, because during post-study questioning they expressed the belief that the preparatory tasks were directly relevant to the experiment's purpose. There were no prospective performance errors in this experiment. None of the participants, across both experiments, retrospectively claimed to have recognized any of the task words during the LDT, thus supporting its use as an indirect test of intention activation. The distribution of data was positively skewed in both Experiments $1 \mathrm{~A}$ and $1 \mathrm{~B}$. To normalize the distributions, reciprocal transformations were performed (cf. Howell, 1992, pp. 315) and statistical analyses were carried out on the transformed values. ${ }^{1}$ Mean scores are presented as units of the untransformed scale.

\section{Experiment 1A}

Initially, the participants' mean response times to the three classes of stimuli (task, nontask, and nonword) were compared (see Table 1 ). A $3 \times 2$ factorial analysis of variance (ANOVA) with item class as the within-subjects factor and group (experimental, control) as the betweensubjects factor yielded a reliable main effect of item class $\left[F(1.56,46.83)=8.56, M S_{\mathrm{e}}=6.843 \times 10^{-8}, p<.01\right]$, but no main effect of group, and no item class $\times$ group interaction (both $F_{\mathrm{s}}<1$ ). Tests of within-subjects repeated contrasts revealed that latencies to nontask items were significantly faster than latencies to nonwords $\left[F(1,30)=22.72, M S_{\mathrm{e}}=1.22 \times 10^{-7}, p<.01\right]$. Latencies to nontask items did not differ from those to task items $(F<1){ }^{2}$

In a subsequent analysis, task words were examined independently and divided according to group (experimental, control) and intentional status (maintained, canceled). As Figure 1 illustrates, a $2 \times 2$ factorial ANOVA with group as the between subjects factor and material as the within-groups factor yielded a reliable interaction $\left[F(1,30)=8.79, M S_{\mathrm{e}}=1.323 \times 10^{-8}, p<.01\right]$. The simple effects revealed that in the experimental group, latencies for intended items were reliably shorter than those

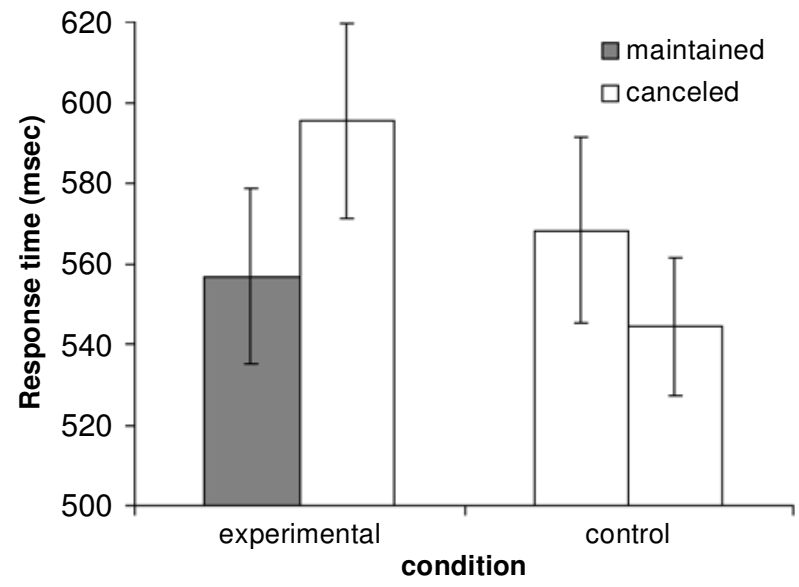

Figure 1. Mean response latencies for maintained and canceled intentions in the experimental and control groups in Experiment $1 \mathrm{~A}$. Bars represent standard errors.

for canceled items $\left[F(1,15)=8.87, M S_{\mathrm{e}}=1.184 \times 10^{-8}\right.$, $p<.01]$. Please recall that both tasks were canceled in the control condition. There was no reliable difference between response times for the two sets of canceled items in the control group $\left[F(1,15)=1.71, M S_{\mathrm{e}}=1.461 \times\right.$ $\left.10^{-8}, p=.210\right]$. Furthermore, there was no reliable difference between response times to maintained items in the experimental condition and response times to either set of canceled items in the control condition $[t(30) \mathrm{s}<1]$.

\section{Experiment 1B}

A $3 \times 2$ factorial ANOVA (item class $\times$ group) was conducted on participants' mean response times to the three classes of stimuli (task, nontask, and nonword). As Table 1 displays, a clear pattern emerged in which responses to task items produced the fastest latencies, responses to nontask items were relatively slower, and the slowest latencies were those to nonwords $\left[F(1.58,72.88)=15.31, M S_{\mathrm{e}}=\right.$ $\left.2.460 \times 10^{-8}, p<.01\right]$. Repeated contrast tests confirmed that response latencies differed significantly between task and nontask item class $\left[F(1,46)=9.25, M S_{\mathrm{e}}=2.071 \times\right.$ $\left.10^{-8}, p<.01\right]$, and also between nontask and nonword item class $\left[F(1,46)=10.21, M S_{\mathrm{e}}=4.113 \times 10^{-8}, p<.01\right]$.

As in Experiment 1 A, latencies to task words were examined as a function of group and intentional status. A

Table 1

Mean Response Latency (in Milliseconds) and Standard Deviations for Task Words, Nontask Words, and Nonwords in the Lexical Decision Tasks in Experiments $1 \mathrm{~A}$ and $1 \mathrm{~B}$

\begin{tabular}{|c|c|c|c|c|c|c|}
\hline \multirow[b]{2}{*}{ Group } & \multicolumn{2}{|c|}{ Task Words } & \multicolumn{2}{|c|}{ Nontask Words } & \multicolumn{2}{|c|}{ Nonwords } \\
\hline & $M$ & $S D$ & $M$ & $S D$ & $M$ & $S D$ \\
\hline \multicolumn{7}{|c|}{ Experiment $1 \mathrm{~A}$} \\
\hline Experimental & 583.90 & 108.20 & 588.92 & 132.62 & 680.18 & 234.62 \\
\hline Control & 557.44 & 94.17 & 572.24 & 107.04 & 643.24 & 113.35 \\
\hline \multicolumn{7}{|c|}{ Experiment 1B } \\
\hline Experimental & 562.17 & 122.78 & 577.93 & 147.08 & 611.61 & 157.94 \\
\hline Control & 560.95 & 87.70 & 595.93 & 119.70 & 664.33 & 252.63 \\
\hline
\end{tabular}


factorial ANOVA (group $\times$ status) revealed a marginally reliable interaction, illustrated in Figure 2, between group and intentional status $\left[F(1,46)=2.91, M S_{\mathrm{e}}=1.652 \times\right.$ $\left.10^{-8}, p=.09\right]$. Repeated contrasts revealed that in the experimental group, as before, latencies to intended items were shorter than those to canceled items $[F(1,23)=$ $\left.6.32, M S_{\mathrm{e}}=1.614 \times 10^{-8}, p=.02\right]$. In the control group, there was no difference between the response times of intended and canceled items $[F(1,23)<1]$. Under these conditions, the intended items were associated, poststudy, with the expectation that these items would be available for referral prior to performance of the task that they described. Finally, latencies to maintained items in the experimental condition did not reliably differ from latencies to maintained items $[t(46)<1]$ or canceled items $[t(46)=1.01, p>.05]$ in the control condition.

\section{DISCUSSION}

The results of two experiments reveal that lexical decisions in response to presentation of task words that are linked to an intact intention to perform an action in the future are faster than those to task words associated with a canceled intention. When both intentions were canceled prior to the LDT, no reliable difference was found between task words (Experiment 1A). Moreover, when an intention coexisted with the expectation that a written copy of the contents would be available, no reliable difference in latencies for these items and canceled items was observed (Experiment 1B). These findings provide a useful extension of work conducted by Goschke and Kuhl (1993), Marsh, Hicks, and Bink (1998), and Marsh et al. (1999). A comparable pattern of results was observed here upon use of a more naturalistic approach that incorporated selfreferent material and self-initiated retrieval conditions.

Marsh, Hicks, and Bink (1998) suggested that heightened activation of intentional constructs might be particularly effective in the absence of external cues to trigger recall and performance. Our findings are consistent with

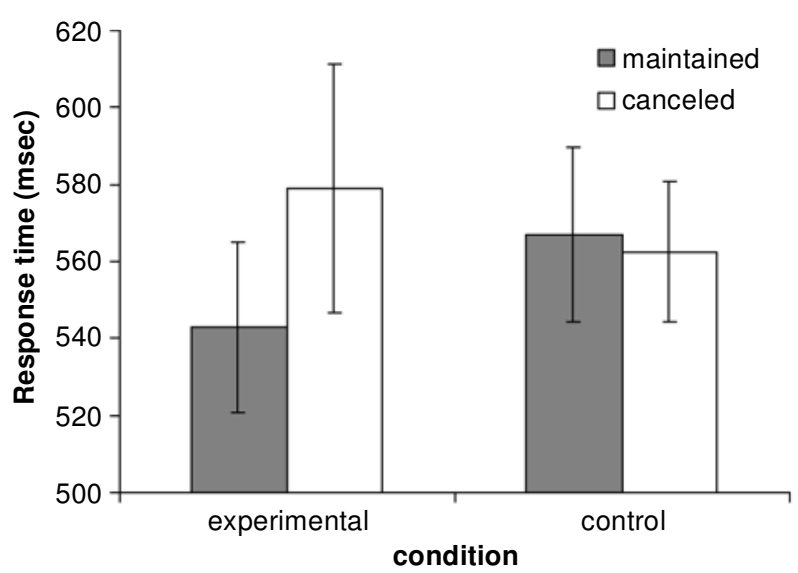

Figure 2. Mean response latencies for maintained and canceled intentions in the experimental and control groups in Experiment 1B. Bars represent standard errors. this conjecture. In the present studies, the need to selfinitiate one's intentions may require increased monitoring of events across the course of the experiment and selfupdating of changes as one approaches an opportunity for intention execution. Both monitoring and updating may interfere with the retrieval or execution of an intention, and in the face of these adversities, heightened activation may increase the probability that it will be carried out. The observation that the ISE occurs not only under event-cued conditions (cf. Goschke \& Kuhl, 1993; Marsh, Hicks, \& Bink, 1998) but also under conditions that require self-initiated retrieval (when it may be most beneficial) provides further support for the claim that intentions have privileged ${ }^{3}$ access in declarative memory (Goschke $\&$ Kuhl, 1993). Moreover, the observation of an ISE under more naturalistic conditions provides some support for claims that the ISE may have some bearing on the relative adeptness with which most people accomplish their everyday intentions. Indeed, Marsh et al. (1999) have observed the ISE under conditions in which people maintain and cancel several unrelated intended actions - a common occurrence in everyday life.

From a theoretical standpoint, interpretations of the ISE have appealed to alternative conceptual accounts. Goschke and Kuhl (1993), for example, argue in favor of the heightened and persistent activation of intentions. In contrast, Marsh, Hicks, and Bink (1998) and Marsh et al. (1999) propose that intentional concepts may be reprocessed more quickly or more efficiently, thus allowing multiple intentions to be activated and deactivated in everyday situations. Furthermore, they observed slower latencies to completed activities and suggested that an inhibitory mechanism might have been responsible for this effect.

Since our participants initially prepared to carry out two activities and then canceled an intention for one of them, Marsh et al.'s (1999) inhibitory account may provide a more parsimonious explanation of the ISE. Whether we are completing or canceling an intention, it may be adaptive to inhibit conditions that lead to intention execution (i.e., we must avoid repeating a completed activity and refrain from executing a canceled intention). Theoretical models are consistent with the latter claim. In Norman and Shallice's (1986) supervisory attentional system, for example, attentional suppression serves to free resources in working memory by the firing of action schemas that laterally inhibit other activated but redundant activities. Other mechanisms also might influence activation of an intended task. In the present study, we have focused on some naturalistic elements of everyday intentions and planned actions, particularly the personal relevance of an intended task and the requirement for self-initiated retrieval. It is feasible that these factors serve to enrich the encoding of intentional constructs and that the qualitative nature of this encoding could heighten the activation of this information.

An important question for future research concerns the relationship between remembering to carry out a delayed intention and the claim that the ISE may aid the accessibility of intended material. Mäntylä’s (1996) retrieval 
sensitivity framework, for example, proposes that the planning for and monitoring of an intended task increases the likelihood that an event, an activity, or a particular time will be noticed, thereby leading to realization of the prospective material. Support for this hypothesis comes from a study in which semantic priming of target categories prior to encoding increased the number of successfully executed intentions (Mäntylä, 1993).

Clearly, the role of the ISE in prospective remembering is an important theoretical and practical question. Current findings, however, allow us to claim only that the ISE is a primed representation, relative to other memory contents, and not that this priming enhances the timely retrieval of prospective constructs. The paradigm employed here, however, might provide a useful means of examining this relationship. The task could be modified by, for instance, increasing both the number of prospective tasks and the attentional demands of the intervening tasks. These two changes would result in participants' having multiple intended representations to contend with and less cognitive resources available for supporting monitoring and self-updating demands. Consequently, the likelihood that an opportunity for prospective performance is realized would be lower and the heightened accessibility of intended actions should be advantageous. If an ISE was more frequently associated with successful realization of intentions under these demanding conditions, a stronger parallel between intention superiority and retrieval sensitivity could be drawn.

In conclusion, the dynamic properties of intended actions have been examined in a paradigm akin to everyday prospective memory. Naturally occurring intentions may be mediated by the personal relevance of the intended activity and by the need for self-initiating retrieval. These factors could contribute to a depth of encoding that either enhances revival rates or sustains the activation of intended activities. The fate of a canceled intention may be regulated by an inhibitory process presided over by the supervisory attentional system (Norman \& Shallice, 1986). Conversely, an intended action representation that is canceled might be deprived of activation from available attentional resources or displaced by stronger associations of an intended activity. In view of these alternative accounts, it will be interesting to examine, more closely, the contribution of facilitatory and inhibitory processes to an intention's status in forthcoming experiments. Moreover, the present paradigm should allow us to explore the extent to which the ISE serves as a precursor for successful remembering.

\section{REFERENCES}

Anderson, J. R. (1993). Rules of the mind. Hillsdale, NJ: Erlbaum. Cattell, R. B., \& CATtell, A. K. S. (1960). Test of " $g$ ": Culture Fair, scale 2, tests $2,3 \& 4$. Champaign, IL: Institute for Personality and Ability Testing.

Dobbs, A. R., \& Reeves, M. B. (1996). Prospective memory: More than memory. In M. A. Brandimonte, G. O. Einstein, \& M. A. McDaniel (Eds.), Prospective memory: Theory and applications (pp. 199-225). Mahwah, NJ: Erlbaum.
ELLIS, J. A. (1996). Prospective memory or the realization of delayed intentions: A conceptual framework. In M. Brandimonte, G. O. Einstein, \& M. A. McDaniel (Eds.), Prospective memory: Theory and applications (pp. 1-22). Mahwah, NJ: Erlbaum.

Francis, W. N., KUČERA, H. \& MACKIE, A. W. (1982). Frequency analysis of English usage: Lexicon and grammar. Boston: Houghton Mifflin.

GoschKe, T., \& KuHL, J. (1993). Representation of intentions: Persisting activation in memory. Journal of Experimental Psychology: Learning, Memory, \& Cognition, 5, 1211-1226.

Howell, D. C. (1992). Statistical methods for psychology (3rd ed.). Boston: PWS-Kent.

KleIN, S. B., \& KinLST Rom, J. F. (1986). Elaboration, organization, and the self-reference effect in memory. Journal of Experimental Psychology: General, 115, 26-38.

Klein, S. B., Loftus, J., \& Burton, H. A. (1989). Two self-reference effects - the importance of distinguishing between self-descriptiveness judgments and autobiographical retrieval in self-referent encoding. Journal of Personality \& Social Psychology, 56, 853-865.

KuHL, J. (1985). Volitional mediators of cognition-behavior consistency: Self-regulatory processes and action versus state orientation. In J. Kuhl \& J. Beckmann (Eds.), Action control: From cognition to behavior (pp. 101-128). Berlin: Springer-Verlag.

MäNTY Lä, T. (1993). Priming effects in prospective memory. Memory, 1, 203-218

MäNTYLä, T. (1996). Activating actions and interrupting intentions: Mechanisms of retrieval sensitization in prospective memory. In M. A. Brandimonte, G. O. Einstein, \& M. A. McDaniel (Eds.), Prospective memory: Theory and applications (pp. 93-113). Mahwah, NJ: Erlbaum.

Marsh, R. L., Hicks, J. L., \& BINK, M. L. (1998). Activation of completed, uncompleted, and partially completed intentions. Journal of Experimental Psychology: Learning, Memory, \& Cognition, 24, 350361 .

Marsh, R. L., Hicks, J. L., \& Bryan, E. S. (1999). The activation of unrelated and canceled intentions. Memory \& Cognition, 27, 320327

Marsh, R. L., Hicks, J. L., \& Landau, J. D. (1998). An investigation of everyday prospective memory. Memory \& Cognition, 26, 633643.

Norman, D. A., \& Shallice,T. (1986). Attention to action: Willed and automatic control of behavior. In R. J. Davison, G. E. Schwartz, \& D. Shapiro (Eds.), Consciousness and self-regulation (pp. 1-18). New York: Plenum.

Rogers, T. B., KuIPER, N. A., \& KirKer, W. S. (1977). Self-reference and the encoding of personal information. Journal of Personality \& Social Psychology, 35, 677-688.

Schaefer, E. G., Kozak, M. V., \& SAgness, K. (1998). The role of enactment in prospective remembering. Memory \& Cognition, 26, 644-650.

Shallice, T., \& Burgess, P. (1991). Deficits in strategy application following frontal lobe damage in man. Brain, 114, 727-741.

\section{NOTES}

1. The same pattern of effects was apparent for the untransformed analysis, although the simple effect resulting from the comparison of intended and canceled items in Experiment $1 \mathrm{~B}$ was only marginally significant $\left[F(1,23)=3.01, M S_{\mathrm{e}}=5,125.08, p=.09\right]$.

2. It should be noted that the results from the present Experiment 1B, Marsh, Hicks, and Bink (1998), and Marsh et al. (1999) revealed a clear pattern in which latencies to task items were reliably faster than those to nontask items. This pattern was not observed in Experiment 1A. One potential reason is that task material was not learned to criterion (in order to preserve naturalistic conditions) and repetition priming may have diminished as a consequence. We like to thank reviewer A for suggesting this possibility.

3. This privileged status is with respect to their "heightened and more sustained level of activation as compared to other memory contents" (Goschke \& Kuhl, 1993, p. 1223). 


\section{APPENDIX A}

Task Materials

(1) Prepare the computer for the next participant

Remove the disk from the drive

Write on your name

Put it in the envelope provided

Push the reset button on the computer's front panel

(2) Prepare the room for the next participant

Take a red booklet from the pile

Place it on the small table

Open the front page

Reverse the sign on the door when you leave

\section{APPENDIX B \\ Room Arrangement and the Layout of Preparatory Task Materials}

Task 1 required the following set-up: a disk with a blank label attached inside the disk drive of the computer, a pen together with a stack of envelopes placed to the right of the computer work station.

Task 2 was arranged as follows: a pile of red booklets stacked to the right of the computer, a small table to the rear of the computer workstation, and a "Testing in Progress" sign on the door.

(Manuscript received December 19, 2000;

revision accepted for publication July 7, 2001.) 